\title{
Campo Eletromagnético e o Fenômeno de Taxia em Cupins de Madeira Seca (Cryptotermes Brevis)
}

\author{
Rodrigo Giovanella ${ }^{1}$, Márcio Pereira da Rocha², Sérgio Luiz Meister Berleze ${ }^{3}$ \\ ${ }^{1}$ Programa de Pós-graduação em Engenharia Florestal, Universidade Federal do Paraná - UFPR, Curitiba/PR, Brasil \\ ${ }^{2}$ Departamento de Engenharia e Tecnologia Florestal, Universidade Federal do Paraná - UFPR, Curitiba/PR, Brasil \\ ${ }^{3}$ Departamento de Física, Universidade Federal do Paraná - UFPR, Curitiba/PR, Brasil
}

\begin{abstract}
RESUMO
Este trabalho teve como objetivo verificar a influência do campo eletromagnético sobre o comportamento de cupins de madeira seca (Cryptotermes brevis), para posterior utilização em tratamento de infestações em peças de madeira. Diferentes cupins foram submetidos a um campo eletromagnético máximo, sob distintas temperaturas. A partir dos resultados, observouse que os cupins são capazes de sentir a presença de um campo eletromagnético e responder a seus estímulos, por meio de taxia negativa (afastamento da fonte do campo) e movimentações de corpo, que se adaptam às variações de campo ao longo da região de exposição, como uma consequência da Lei de Faraday de indução magnética e da hipótese ferromagnética, a qual se baseia na presença de partículas magnéticas no organismo que funcionam como sensores de campos. O campo eletromagnético fez, ainda, com que os cupins tivessem descontrole de segmentos e de membros acessórios.
\end{abstract}

Palavras-chave: indução magnética, taxia negativa, descontrole do corpo.

\section{Electromagnetic Field and the Phenomenon of Taxis in Termites (Cryptotermes Brevis)}

\begin{abstract}
This paper aimed to verify the influence of the electromagnetic field on the behavior of drywood termites (Cryptotermes brevis) for subsequent use in treating infestations of timber pieces. Different termites were subjected to a maximum electromagnetic field under different temperatures. The results showed that termites are able to sense the presence of an electromagnetic field and respond to its stimuli by means of negative taxis (removal of the field source) and body movements to suit field variations over the area of exposure, as a consequence of Faraday's law of magnetic induction and ferromagnetic hypothesis, which are based on the presence of magnetic particles in the body which act as field sensors. Also, the electromagnetic field caused the termites to present uncontrolled segments and accessory limbs.
\end{abstract}

Keywords: magnetic induction, negative taxis, uncontrolled body. 


\section{INTRODUÇÃO}

A madeira é um material que, devido a suas características, esteve presente desde os primórdios do desenvolvimento da civilização humana. Propriedades, como a resistência mecânica elevada em relação à massa própria, a facilidade de usinagem, as boas propriedades de isolamento térmico e elétrico, e, inclusive, a possiilidade de ser encontrada com uma ampla faixa de texturas e colorações, garantiram a este material um lugar de destaque nas mais diversas indústrias e utilizações (Lepage, 1986; Rocha, 2000; Giovanella, 2009). Entretanto, a madeira apresenta uma vida útil pequena em dadas condições, com sua durabilidade comprometida por uma série de fatores físicos, químicos e/ou biológicos. Isto ocorre devido à sua natureza orgânica, em termos de constituintes poliméricos, sendo os principais a celulose e as hemiceluloses (Lepage, 1986; Rocha, 2001). Deste modo, a tecnologia de preservação de madeiras destaca-se como fundamental para a utilização deste material, sendo seu objetivo inicial aumentar a durabilidade da madeira e, com isso, incentivar o uso da mesma. Outros pontos são considerados como chaves também na preservação, como, por exemplo, evitar a migração de espécies biológicas para ambientes não naturais, por intermédio das exportações.

Este trabalho tem como hipótese: um campo eletromagnético é capaz de interagir com o organismo de cupins de madeira seca, de forma a provocar taxia nos indivíduos expostos, assim afastando ou repelindo os insetos de uma determinada área ou de uma peça de madeira. Deste modo, dependendo da metodologia de utilização, o campo eletromagnético pode ser considerado para tratamentos curativos e preventivos. Este método é inerte à matéria e não apresenta riscos de contaminação, como os químicos e/ou os de irradiação (ionizante). Assim, o objetivo do presente trabalho foi verificar a influência do campo eletromagnético sobre o comportamento de cupins de madeira seca (Cryptotermes brevis).

\section{MATERIAL E MÉTODOS}

\subsection{Local do experimento}

Os ensaios de prova foram todos realizados em um ambiente especialmente adaptado para as condições dos testes, que incluíram a mínima interferência eletromagnética externa e condições de clima controlado (descrito em 2.4.2 - Teste de sensibilidade ao campo eletromagnético). A sala de testes, com área de $1,56 \mathrm{~m}^{2}(1,20 \times 1,30)$, tinha sua estrutura composta de material dry wall e localizavase na região de Curitiba, Paraná.

\subsection{Eletroímã - estrutura e composição}

O eletroímã foi construído a partir de uma bobina com 300 espirais, utilizando-se um fio de cobre encapado de $25 \mathrm{~mm}^{2}$ de seção transversal e um núcleo composto por vergalhões de ferro envernizados, com $10 \mathrm{~mm}$ de diâmetro, colocados no interior de um tubo de PVC, com $10 \mathrm{~cm}$ de raio e 18 $\mathrm{cm}$ de comprimento. Os vergalhões foram escolhidos devido ao pequeno diâmetro, sendo posteriormente envernizados, com o intuito de minimizar os efeitos das Correntes de Foucault (Figura 1).

A fonte de alimentação foi a rede de distribuição de energia de Curitiba, com tensão nominal de $127 \mathrm{~V}$ e frequência de $60 \mathrm{~Hz}$, sendo estes valores
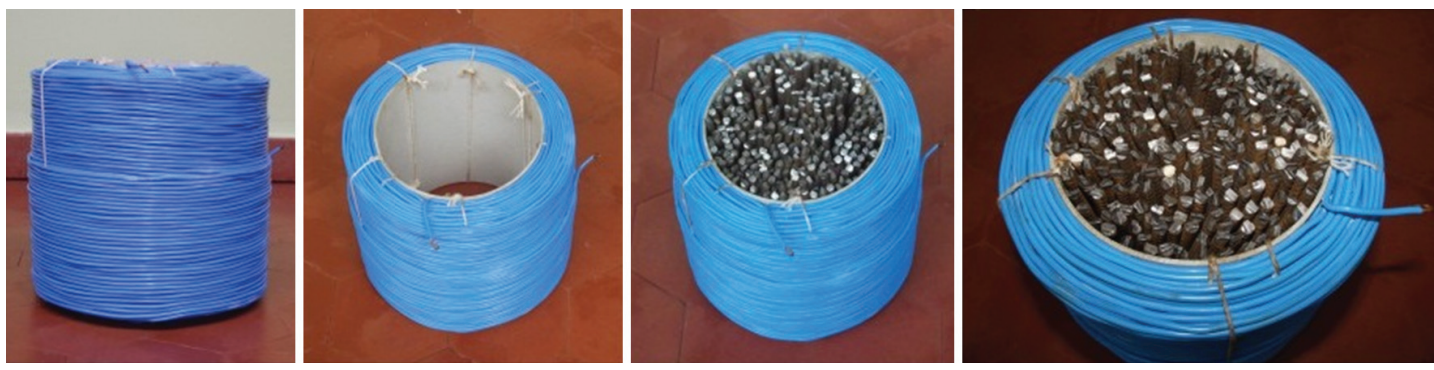

Figura 1. Bobina do eletroímã construído.

Figure 1. Coil of the electromagnet built. 
escolhidos devido à facilidade de disponibilidade para aplicações posteriores. No circuito do eletroímã, foram colocadas em série seis lâmpadas, como resistências limitadoras, para evitar que uma corrente muito intensa circulasse pela bobina. Estas lâmpadas também foram inseridas no experimento de forma que indicassem o funcionamento do equipamento e permitissem o controle $\mathrm{da}$ intensidade $\mathrm{da}$ corrente e do campo eletromagnético. As lâmpadas foram colocadas em paralelo umas com as outras, permitindo o funcionamento independente entre si. Também, por critérios de segurança, foi inserido no circuito um disjuntor de $10 \mathrm{~A}$.

Para a realização dos testes, o eletroímã foi fixado no interior de uma caixa, com dimensões de $70 \times 70$ $\times 80 \mathrm{~cm}$, tendo cinco de suas superfícies compostas por madeira reconstituída (MDF) e uma de vidro, para facilitar as observações (Figura 2).

$\mathrm{O}$ intuito da caixa de teste foi delimitar uma área para estudo, na qual havia a atuação do campo eletromagnético, sendo possível, assim, descrever as linhas de campo magnético na região e sua forma de atuação. Outra questão relevante foi o controle das condições internas da caixa de teste, permitindo uma condição favorável aos cupins, garantindo-lhes umidade relativa adequada e proteção contra luz, nos períodos de não observação.

\subsection{Espécie de inseto xilófago estudado}

Os agentes biodeterioradores escolhidos para a execução deste trabalho foram os cupins da família Kalotermitidae, gênero Cryptotermes, espécie Cryptotermes brevis (Walker, 1853), provenientes de uma colônia situada na região de Curitiba, Paraná. Foram escolhidos por ser uma espécie recorrente na região, auxiliando a coleta e indo ao encontro com a realidade local de infestações.

\subsection{Testes de comportamento}

Para a determinação dos efeitos de um campo eletromagnético sobre os insetos estudados, foram realizados dois tipos de ensaios. Estes testes tiveram o objetivo de analisar o comportamento dos cupins sem a influência do campo eletromagnético e, posteriormente, com a influência deste campo, de forma a determinar a sensibilidade dos indivíduos.

\subsubsection{Teste de padrão de comportamento dos cupins}

Este teste buscou determinar um padrão de comportamento para os insetos, de modo que se pudessem avaliar os efeitos causados pelo campo eletromagnético no grupo e de forma individual.

Para estimar este padrão de comportamento, foi utilizada a metodologia apresentada por Giovanella (2009), em seu Teste de Caracterização do Comportamento 'Natural' dos Cupins, adequando-a às condições do teste deste trabalho. Assim, foram consideradas duas amostras, com seis indivíduos cada, as quais foram observadas durante um período de 24 horas. Na primeira hora, foram feitas observações de cinco em cinco minutos, e na segunda e na terceira horas, em intervalos de dez minutos. Da quarta à oitava hora, as amostras foram observadas a cada hora e, na nona hora, de dez em dez minutos. Nas 14 horas seguintes, foram
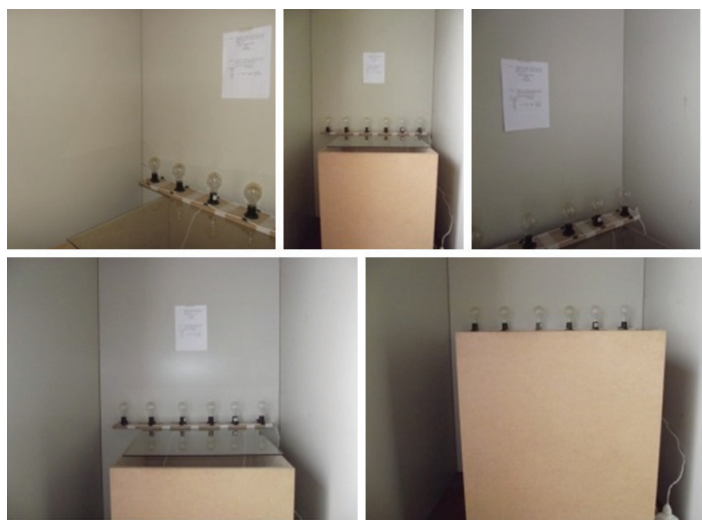
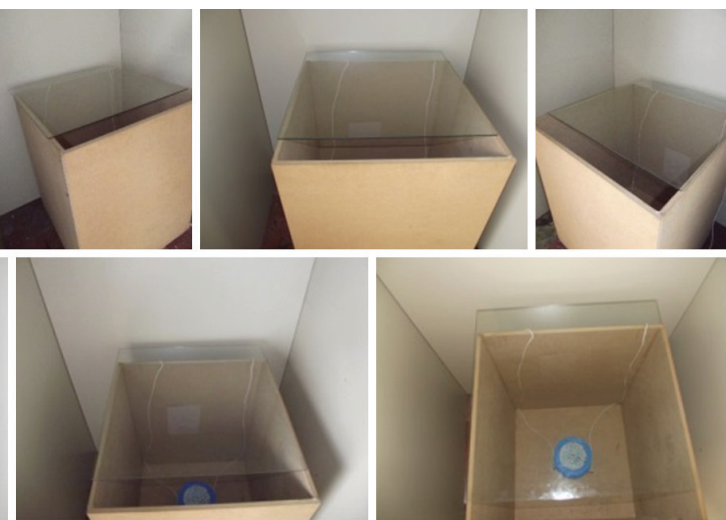

Figura 2. Caixa de teste vista de vários ângulos.

Figure 2. Test box view from various angles. 
realizadas observações de duas em duas horas, e, posteriormente, completando o período de tempo estipulado, foi realizada uma observação na $24 .^{a}$ hora.

Foram adotadas duas metodologias de observação, na sequência do teste, para a classificação do comportamento dos insetos estudados. A primeira, com o intuito de analisar os indivíduos dentro do grupo amostral, seguiu conforme o modelo de teste (Giovanella, 2009), sendo a classificação do comportamento padrão dividida em parados, lento/moderado ou acelerado/agitado. Em cada observação, os insetos foram classificados conforme um dos tipos de atividades descritas. Isto foi realizado para que se pudesse construir uma curva de atividade dos insetos, permitindo estimar um comportamento padrão para os mesmos. As observações foram realizadas apenas por um pesquisador treinado, para minimizar as interferências de interpretações dos padrões de movimento.

A segunda metodologia, com o objetivo de analisar os efeitos individuais em cada inseto, seguiu o padrão de comportamento apresentado na Tabela 1. A verificação da forma como os indivíduos se movimentavam - não apenas em termos de deslocamento, mas também na maneira de interagir com o meio - foi imprescindível para avaliar os possíveis efeitos do campo eletromagnético.
As observações foram realizadas respeitando as condições do experimento, levando em consideração a presença do eletroímã e demais particularidades, como temperatura e umidade. Estes detalhes estão descritos no item 'Teste de sensibilidade ao campo eletromagnético.

\subsubsection{Teste de sensibilidade ao campo eletro- magnético}

O objetivo deste teste foi verificar como o campo eletromagnético poderia afetar o organismo dos insetos. Foi considerada a variação de temperatura do meio para potencializar os efeitos do campo eletromagnético, a partir da alteração do potencial elétrico biológico. Assim, definiram-se cinco classes de temperaturas, sendo a primeira uma temperatura padrão ambiente, definida através da temperatura média anual, num período de cinco anos (de janeiro de 2006 a outubro de 2010), da cidade de Curitiba, Paraná. As outras quatro classes foram definidas com base na temperatura média padrão, sendo duas em limites próximos e duas em limites extremos. Utilizou-se a umidade relativa média, também num período de cinco anos (de janeiro de 2006 a outubro de 2010) da cidade de Curitiba, Paraná, para todas as temperaturas avaliadas, conforme Tabela 2 .

Tabela 1. Classificação das atividades e comportamento dos cupins Cryptotermes brevis (Isoptera: Kalotermitidae) - Método individual.

Table 1. Classification of activities and behavior of termites Cryptotermes brevis (Isoptera: Kalotermitidae) - Alone method.

\begin{tabular}{|c|c|c|c|c|}
\hline \multicolumn{5}{|c|}{ Atividade e comportamento dos cupins } \\
\hline \multirow{2}{*}{$\begin{array}{c}\text { Parte do Segmento } \\
\text { Analisada }\end{array}$} & \multicolumn{4}{|c|}{ Efeito Associado } \\
\hline & 1 & 2 & 3 & 4 \\
\hline \multicolumn{5}{|l|}{ Cabeça } \\
\hline Tórax & \multirow{4}{*}{ Imóvel } & \multirow{4}{*}{ Movimentos Lentos } & \multirow{4}{*}{ Movimentos Rápidos } & \multirow{4}{*}{$\begin{array}{c}\text { Movimentos } \\
\text { Descontrolados } \\
\text { (ato de tremer) }\end{array}$} \\
\hline Abdômen & & & & \\
\hline Antenas & & & & \\
\hline Pernas & & & & \\
\hline
\end{tabular}

Tabela 2. Temperatura $\left({ }^{\circ} \mathrm{C}\right)$ e Umidade Relativa (\%) dos testes de sensibilidade ao campo eletromagnético. Table 2. Temperature $\left({ }^{\circ} \mathrm{C}\right)$ and relative humidity $(\%)$ of sensitivity tests to the electromagnetic field.

\section{Classe de Temperatura}

Temperatura Padrão

1

2
Temperatura $\left({ }^{\circ} \mathrm{C}\right)$

18

11

25

0

50
Umidade Relativa (\%)

84

84

84

84

84 
Foram retiradas, por amostragem aleatória simples, 25 amostras com quatro indivíduos cada, os quais foram expostos ao campo eletromagnético nas diferentes classes de temperatura. Para cada classe de temperatura, foram realizadas cinco repetições, em razão do número reduzido de indivíduos por amostra. Foram escolhidos quatro indivíduos por amostra, por duas razões principais: a primeira, devido à atenção necessária para avaliar, nos insetos, de maneira individual e em cada segmento, o efeito do campo eletromagnético; a segunda, em função de que os insetos foram coletados de uma única colônia e este número, quatro, foi definido a partir do tamanho da colônia e do número de testes realizados.

O tempo de exposição ao campo eletromagnético foi de 60 minutos ininterruptos, sendo que o mesmo inseto não foi exposto a mais de uma classe de temperatura. A intensidade utilizada para o campo eletromagnético, para todos os testes, foi a máxima, segundo as propriedades do eletroímã construído.

As observações ocorreram em intervalos de cinco minutos, iniciando-se no momento em que os insetos foram expostos ao campo eletromagnético. Após a exposição ao campo eletromagnético, foram feitas observações em intervalos de uma hora, num período total de 24 horas, e mais duas observações, realizadas 48 e 72 horas após o teste.

\section{RESULTADOS E DISCUSSÃO}

No exato momento em que o eletroímã foi ligado, tempo de zero minuto de observação, os cupins, nas cinco repetições, alteraram seu comportamento imediatamente, apresentando três principais efeitos; (1) percepção do campo eletromagnético pelos cupins, (2) taxia negativa e (3) descontrole do tórax e abdômen.

Para o primeiro efeito, os cupins apresentaram um alinhamento do corpo de todos os insetos em conjunto com uma orientação voltada para a superfície frontal da caixa de teste (Figura 3). Ao mesmo tempo, as antenas passaram a apresentar um comportamento associado ao nível três de atividade, considerada como rápida e agitada. Estes dois fenômenos, de alinhamento e orientação, sugerem que os cupins foram capazes de perceber variações no campo eletromagnético e de responder a estes estímulos.

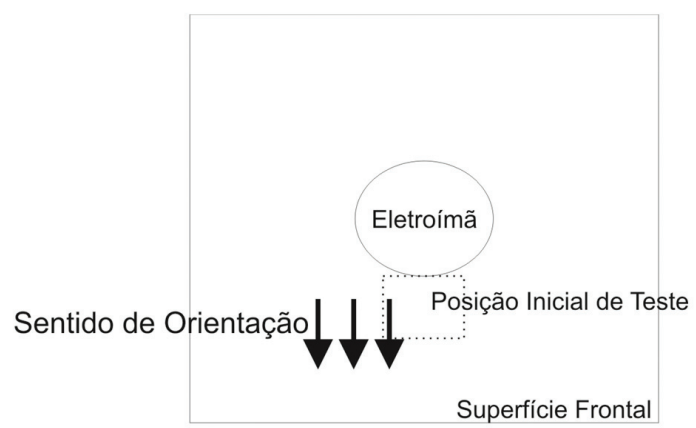

Figura 3. Diagrama da caixa de teste e sentido de orientação dos cupins da espécie Cryptotermes brevis no momento em que o eletroímã foi ligado - tratamento à temperatura padrão.

Figure 3. Diagram of the test box and sense of direction of termites Cryptotermes brevis when the electromagnet has been switched on - the standard temperature treatment.

Abraçado (2006) comenta em seu trabalho que os cupins são influenciados pelo campo geomagnético, enfatizando que estes insetos têm a capacidade de perceber as variações magnéticas ao seu redor. Além de Abraçado (2006), outros autores, como Esquivel et al. (2007), Hsu C-Y et al. (2007) e Wajnberg et al. (2010), discorreram sobre a existência de um magnetorreceptor, baseandose no fato de que os insetos utilizam os campos magnéticos ao seu redor para se orientar. Ou seja, os insetos têm a capacidade de perceber e interpretar os sinais magnéticos, usando esta informação para se locomoverem nas proximidades de suas respectivas colônias ou mesmo em movimentos migratórios. Segundo Wajnberg et al. (2010), a magnetorrecepção é uma habilidade sensorial de perceber os sinais ou estímulos magnéticos, efetuar sua transdução e transferi-las para o sistema nervoso e o cérebro, em que ocorre o processamento e a interpretação da informação. Abraçado (2006) ressaltou que este mecanismo de magnetorrecepção é um mecanismo de orientação ativa que ocorre em animais que possuem sistema nervoso, tendo um funcionamento mais sofisticado que envolve a detecção do campo e as subsequentes etapas, apresentadas por Wajnberg et al. (2010) e descritas acima. Poucos estudos foram feitos até o momento e apenas algumas hipóteses existem acerca do funcionamento destes sensores. 
Uma destas hipóteses está baseada na Lei de Faraday de indução magnética, segundo a qual se supõe que o organismo detecta uma diferença de potencial elétrico fraca, gerado na superfície do seu corpo ou em suas fibras nervosas, resultantes de seu movimento através do campo magnético. Este potencial se elevaria de forma linear com o campo e a velocidade de deslocamento do inseto (Abraçado, 2006). O autor explica que o funcionamento desta hipótese está ligado ao mecanismo de ressonância de íon paramétrico, tendo como partida a ideia de que o sensor magnético é um íon ligado a uma proteína. Desta forma, um campo magnético pode causar vibrações neste íon dentro da cadeia proteica, levando a alterações nas ligações com o íon de cálcio. Ressalta-se que isto pode afetar os terminais nervosos bioquímicos devido à despolarização da membrana celular e consequente ativação da célula excitada. $\mathrm{O}$ autor ainda comenta outro modelo que se baseia em reações de radicais livres alteradas por campos magnéticos. Nestes dois modelos, o efeito de ressonância, em nível atômico ou molecular, é induzido pela combinação de campos magnéticos estáticos e oscilantes.

Outra hipótese apresentada por Abraçado (2006) e amplamente discutida por Wajnberg et al. (2010) é a ferromagética. Esta teoria se baseia no princípio de que existem partículas magnéticas presentes no organismo e que estas funcionam como sensores de campos. A ideia é sustentada pela descoberta de magnetita em várias espécies de animais, incluindo os insetos (Abraçado, 2006). Wajnberg et al. (2010) Esses autores comentam ainda que a resposta do processo de magnetorrecepção depende diretamente do tamanho da partícula magnética envolvida no processo.

A partir destas informações, pode-se concluir que os cupins testados, independentemente da repetição, foram capazes de perceber, de maneira muito rápida, as variações no campo magnético no interior da caixa de teste e que sua orientação e mobilidade das antenas foram respostas diretas a este estímulo.

O segundo principal efeito observado no momento em que o eletroímã foi ligado veio a confirmar a percepção e a resposta, a partir de um órgão sensorial, dos cupins ao estímulo do campo magnético. Este efeito foi uma taxia negativa em nível moderado, ou seja, afastamento do eletroímã com uma movimentação variando de lenta a moderada, de acordo com a classificação de movimento apresentada na Tabela 1. Esta movimentação foi feita em direção à face frontal da caixa de teste e concentrada à esquerda da mesma, conforme Figura 4.

Todos os insetos submetidos ao tratamento apresentaram uma tendência de afastamento do eletroímã. Houve momentos em que os cupins apresentaram este padrão simultaneamente e outros momentos em que alguns se movimentavam em taxia negativa e os demais se apresentavam imóveis, em termos de deslocamento; entretanto, estes imóveis apresentavam-se com grande agitação em todo o corpo, sendo a antena verificada em alta atividade em todos estes cupins. Uma possível explicação seria o posicionamento e a orientação inicial destes no interior da caixa de teste em relação ao eletroímã que, por intermédio da Lei de Indução de Faraday e da intensidade do campo eletromagnético, pode ter influenciado de forma mais significativa os sentidos e percepções dos insetos.

O terceiro efeito observado foi o descontrole do tórax e do abdômen, sendo este fenômeno verificado em 17 indivíduos dentre as repetições, ao longo do período de observação. Estes indivíduos exibiram seus tórax e abdômens de forma trêmula, agitada e aparentemente fora do controle de coordenação

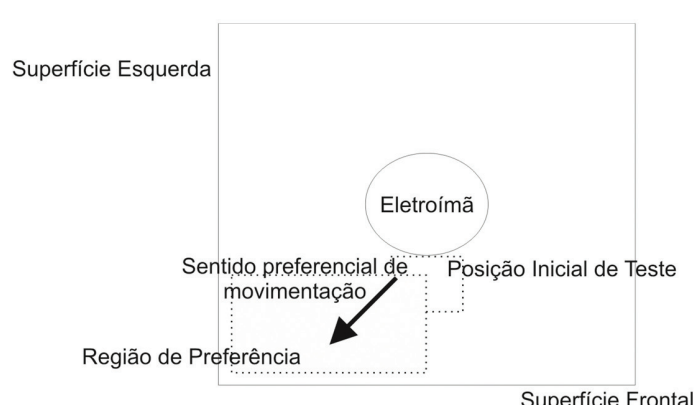

Figura 4. Sentido de orientação e início de movimentação dos cupins da espécie Cryptotermes brevis no momento em que o eletroímã foi ligado, para o tratamento à temperatura padrão - primeira repetição.

Figure 4. Direction of orientation and initiation of movement of termites Cryptotermes brevis when the electromagnet was switched to the treatment temperature pattern - first repeating. 
motora e proprioceptivo natural, dando a impressão de terem sido atingidos por alguma força externa. Para este efeito visualizado, existem duas possibilidades que não se excluem mutuamente, sendo, inclusive, esse fato passível de que ambos os efeitos tenham ocorrido conjuntamente.

A primeira possível causa deste efeito está relacionada ao campo magnético. De acordo com Abraçado (2006), a presença de duas partículas, em diferentes composições e tamanhos, tem sido considerada no organismo dos insetos, sendo estas a magnetita e a maghemita. Savian (2009) descreveu estes dois minerais como de origem orgânica e comentou que a entrada destes no organismo dos insetos ocorre por meio da dieta e do efeito de biomineralização.

Os resultados apresentados por esse autor demonstraram que, nos cupins testados, não foi possível determinar a presença de magnetita, mas identificou-se a presença de titanomagnetita e/ ou maghemita. Esquivel et al. (2004) relataram nanopartículas com parâmetros magnéticos comparáveis aos da magnetita em cupins Neocapritermes opacus. Embora a origem desses minerais fosse inicialmente incerta, estudos subsequentes de Oliveira et al. (2008) revelaram que os próprios cupins sintetizam as partículas ferrimagnéticas.

Segundo Oliveira (2005), medidas de microscopia eletrônica indicaram a presença de magnetita biogênica em duas espécies de cupins, Nasutitermes exitiosus e Amitermes meridionalis. $\mathrm{O}$ autor ainda relatou que medidas de magnetometria indicaram a presença de pequenas concentrações de material magnético, com maior quantidade presente no tórax e abdômen, quando comparados à cabeça, fato este que justifica uma maior interação e descontrole nestes segmentos frente ao campo eletromagnético aplicado.

Abraçado (2006) ressaltou que a presença destes minerais não ocorre de forma homogênea ao longo do corpo dos insetos e que suas presenças sugerem componentes diamagnéticas e/ou paramagnéticas, com diferentes contribuições de acordo com suas concentrações. O autor apresentou resultados, para formiga Solenopsis interrupta, que revelaram que o material biológico encontrado no tórax e nas antenas teve uma componente diamagnética, enquanto que, no abdômen, esta componente é predominantemente paramagnética, que pode ser resultante do material magnético ingerido. De acordo com Halliday et al. (1995) e Nussenzveig (2007), o diamagnetismo é uma manifestação da Lei de Indução de Faraday atuando sobre os elétrons atômicos, cujos movimentos são equivalentes a minúsculas espiras de corrente. Segundo os mesmos autores, materiais diamagnéticos não possuem dipolos magnéticos intrínsecos, mas é possível induzir dipolos magnéticos nestes materiais pela ação de campos magnéticos externos.

Abraçado (2006) comentou que a componente aparentemente diamagnética se sobrepõe à histerese ferro(i)magnética, levando à ideia de que este efeito diamagnético foi superior. Isto sugere que, no tórax dos cupins expostos ao campo eletromagnético do presente trabalho, houve uma reação magnética, desencadeada pelo fenômeno de magnetização, que originou momento de dipolo magnético nos minerais presentes em sua estrutura.

Em relação ao abdômen, o efeito paramagnético teve uma dinâmica semelhante; entretanto, os materiais paramagnéticos apresentam momento de dipolos magnéticos intrínsecos que, na ausência de um campo externo, ficam orientados em direções distribuídas ao acaso (Nussenzveig, 2007; Halliday et al., 1995). Deste modo, quando o campo magnético gerado pelo eletroímã passou a atuar na área em que os insetos estavam presentes, o momento de dipolo magnético dos minerais presentes na estrutura dos insetos sofreu uma tendência ao alinhamento com o campo externo, fenômeno este que pode intensificar o campo (Halliday et al., 1995).

Outro ponto importante para o efeito paramagnético são os metais alcalinos, sódio e potássio, que estão presentes na composição da estrutura celular (Aires, 2008). O balanço químico destes compostos determina o funcionamento regular da célula e seu desequilíbrio pode alterar o valor do potencial de ação da célula, por meio da variação da permeabilidade da membrana. Isto pode, num primeiro momento, forçar o funcionamento da célula, que responderá com uma compensação de entrada ou saída de sódio ou potássio na mesma, desencadeando o processo de transporte ativo. Isto leva a um aumento na demanda energética e 
sobrecarrega o sistema nervoso, quando associado aos efeitos do campo elétrico. Com base no exposto, pode-se dizer que estes efeitos, diamagnéticos e paramagnéticos, foram sentidos pelos cupins testados e tiveram como consequência uma reação adversa nos seus segmentos, por meio de uma sensibilização fisiológica, que culminou no descontrole e na ação de tremor.

A segunda causa para o efeito observado nos insetos deste trabalho está relacionada ao campo elétrico. De acordo com Kaune (1993), qualquer corpo condutor, incluindo organismos vivos, pode sofrer perturbações oriundas da presença do campo elétrico. Esta perturbação ocorre em consequência de o campo aplicado, E, induzir uma densidade de carga elétrica sobre a superfície do corpo exposta, que acaba por gerar um segundo campo elétrico, E'. O autor explica que o campo elétrico total é a soma dos campos, E e E', e que, no interior do corpo, são praticamente iguais em magnitude, mas com direções opostas, portanto, normalmente, anulando-se.

Entretanto, fora do corpo, os campos, E e E', podem se somar positivamente, resultando em uma intensidade maior, em vez de se anularem. Assim, este campo aumentado tende a ser maior na superfície externa e, principalmente, em regiões curvas do corpo. Como indicado por Kaune (1993), o campo elétrico externo aplicado sobre um organismo gera diferentes potenciais no mesmo (Figura 5), induzindo estímulos, ou impulsos, elétricos nas células mais afetadas.

Ao considerar que o campo magnético, por intermédio da influência nos íons de sódio, altera o potencial elétrico da membrana celular, os impulsos elétricos gerados pela presença do campo elétrico podem fazer com que as células sejam ativadas mesmo que em uma situação inicial considerada de repouso da mesma. Ou seja, considerando-se o tecido muscular que está mais externo ao corpo dos insetos, pode-se visualizar a ativação destas estruturas mesmo que o inseto não o faça de forma consciente ou controlada. Assim, contrações involuntárias podem afetar as relações de equilíbrio e propriocepção dos insetos, fazendo com que percam a capacidade de perceberem a si, prejudicando sua mobilidade e deslocamento, e ao ambiente, também em relação ao deslocamento, mas principalmente sobre sua segurança, atrapalhando sua percepção a predadores ou estímulos adversos.

Outro efeito visualizado, ao longo do período de observação, foi a alteração de movimento, em que 16 dos 20 insetos permaneceram imóveis

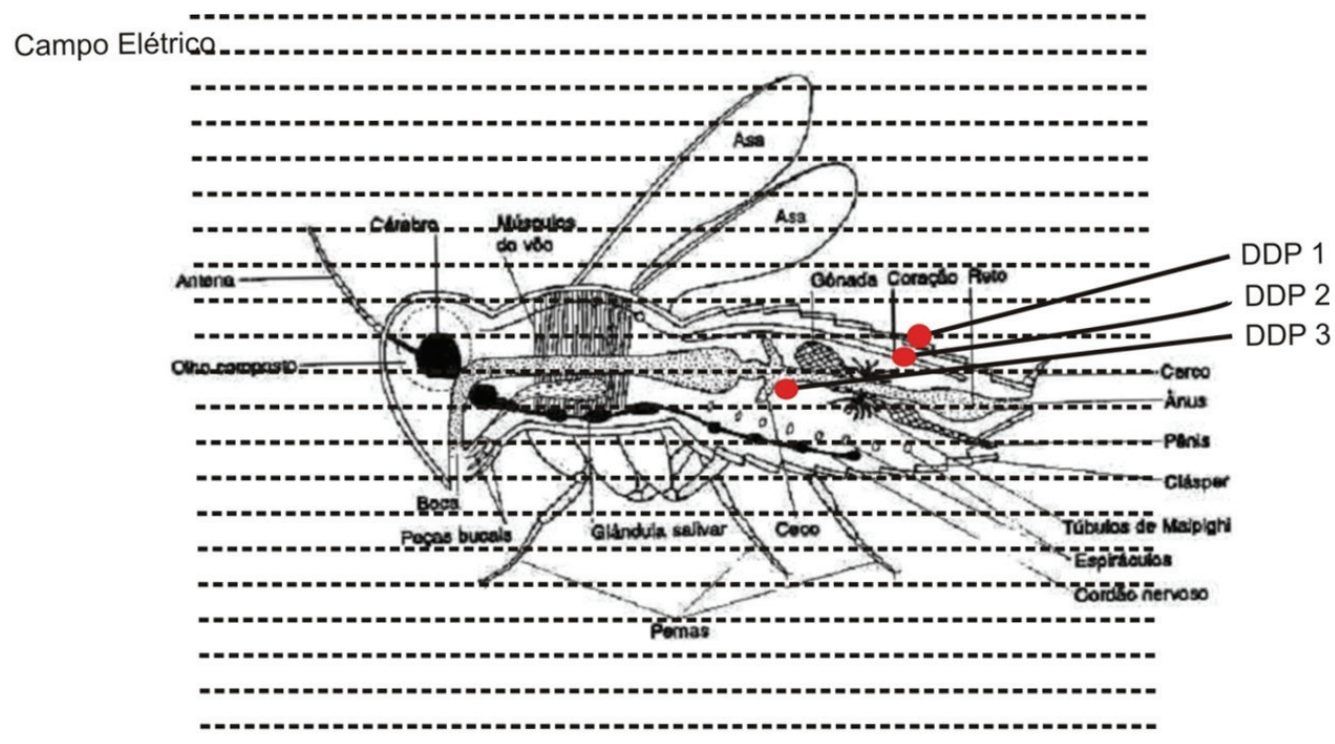

Figura 5. Diferença de potenciais elétricos (DDP) no interior do corpo de um inseto. DDP $1>$ DDP $2>$ DDP 3 . Fonte: Adaptado de Leite (2011).

Figure 5. Difference in electrical potential (DDP) within the body of an insect. DDP 1> DDP 2> DDP 3. Source: Adapted from Leite (2011). 
ou em movimentação considerada não natural. De acordo com o observado, parte dos insetos, nos diferentes tempos de análise, encontrava-se totalmente imóvel, com exceção de suas antenas, que exibiram um comportamento agitado. Estes indivíduos encontravam-se na região de preferência (Figura 4), assim como os outros que permaneceram em movimento de deslocamento e segmentos extremamente agitados. Alguns dos cupins moveramse de forma a fazer um movimento ondulatório contínuo com seu corpo, como o movimento de uma serpente, sendo este efeito visualizado em repetições diferentes e recorrentes nas diversas observações. Esses cupins faziam breves pausas e logo reiniciavam esta tendência de movimento.

Outros cupins ficaram desorientados, com movimentos intermitentes de taxia positiva e negativa variando sempre nas mesmas posições. Estes comportamentos podem estar vinculados com a hipótese de os magnetorreceptores funcionarem com base na Lei de Faraday de indução magnética. De acordo com Abraçado (2006), e conforme discutido anteriormente, a diferença de potencial elétrico gerada na superfície do corpo do cupim, prevista por Kaune (1993), é uma resultante diretamente dependente do seu deslocamento, em termos de velocidade e forma. Ou seja, a maneira como os cupins se moveram, serpenteando o corpo, foi uma resposta ao estímulo do campo eletromagnético, que influenciou suas percepções, buscando uma melhor adaptação a este novo fator ambiental ao qual estavam expostos. Da mesma forma, a imobilidade dos outros cupins pode ter sido uma resposta de adaptação a este estímulo do campo induzido. Assim, a desorientação de alguns insetos demonstrou que estes foram mais sensíveis ao estímulo e que não conseguiram uma boa resposta de adaptação ao meio.

\section{CONCLUSÕES}

De acordo com os resultados obtidos, pode-se concluir que;

- Os cupins Cryptotermes brevis são capazes de perceber a presença de um campo eletromagnético e responder a seus estímulos;

- O campo eletromagnético provoca, independentemente da temperatura, taxia negativa nos cupins Cryptotermes brevis;
- O campo eletromagnético provocou perda de controle de segmentos e membros acessórios, fazendo com que os cupins Cryptotermes brevis perdessem a capacidade de executar suas ações naturais.

\section{STATUS DA SUBMISSÃO}

Recebido: 14 nov., 2013

Aceito: 10 jun., 2014

Publicado: 21 nov., 2014

\section{AUTOR(ES) PARA CORRESPONDÊNCIA}

\section{Rodrigo Giovanella}

Programa de Pós-graduação em Engenharia

Florestal, Universidade Federal do

Paraná - UFPR, CEP 80210-170, Curitiba, PR,

Brasil

e-mail: giggio_giovanella@yahoo.com.br

\section{REFERÊNCIAS}

Abraçado LG. Caracterização de material magnético em formigas Solenopsis interrupta: magnetometria SQUID e Ressonância Ferromagnética [tese]. Rio de Janeiro: Centro Brasileiro de Pesquisas Físicas; 2006. PMid:17071652 PMCid:PMC1751416

Aires MM, editor. Fisiologia. 3 ed. Rio de Janeiro: Guanabara Koogan; 2008. PMCid:PMC2570821

Esquivel DMS, Wajnberg E, Cernicchiaro GR, Alves OC. Comparative magnetic measurements of migratory ant and its only termite prey. Journal of Magnetism and Magnetic Materials 2004; 278: 117-121. http://dx.doi. org/10.1016/j.jmmm.2003.12.327

Esquivel DMS, Wajnberg E, Nascimento FS, Pinho MB, Lins de Barros HGP, Eizemberg R. Do geomagnetic storms change the behaviour of the stingless bee guiruçu (Schwarziana quadripunctata)? Naturwissenschaften 2007; 94(2):139-142.

Giovanella R. Uso de Frequências Sonoras no Controle de Cupins de Madeira Seca Cryptotermes brevis (Isoptera: Kalotermitidae) [dissertação]. Curitiba: Universidade Federal do Paraná; 2009.

Halliday D, Resnick R, Walker J. Fundamentos de Física 3 - Eletromagnetismo. 4 ed. Rio de Janeiro: Editora Livros Técnicos e Científicos; 1995.

Hsu C-Y, Ko F-Y, Li C-W, Fann K, Lue J-T. Magnetoreception System in Honeybees (Apis mellifera). PLoS One 2007; 2(4): e395. PMid:17460762 PMCid:PMC1851986. http://dx.doi.org/10.1371/ journal.pone.0000395 
Kaune WT. Introduction to Power-frequency Electric and Magnetic Fields. Environmental Health Perspectives 1993 December; 101(Suppl 4): 73-81. PMid:8206045 PMCid:PMC1519708. http://dx.doi.org/10.1289/ ehp.93101s473

Leite GLD. Entomologia Básica. Montes Claros: Universidade Federal de Minas Gerais; 2011. Material Didático.

Lepage ES, editor. Manual de Preservação de Madeiras. São Paulo: IPT; 1986. vol. I.

Nussenzveig HM. Curso de Física BásicaEletromagnetismo. 6. ed. São Paulo: Editora Blucher; 2007. vol. 3.

Oliveira JF, Alves OC, Esquivel DMS, Wajnberg E. Ingested and biomineralized magnetic materials in the prey Neocapritermes opacus temite: FMR characterization. Journal of Magnetic Ressonance 2008; 191: 112-119. PMid:18194874. http://dx.doi. org/10.1016/j.jmr.2007.12.006

Oliveira JF. Caracterização do Material Magnético do Cupim Neocapritermes opacus: Magnetometria SQUID e Ressonância Ferromagnética [dissertação]. Rio de Janeiro: Centro Brasileiro de Pesquisas Físicas; 2005.

Rocha MP. Biodegradação e Preservação da Madeira. Curitiba: Fupef; 2001. Série Didática.

Rocha MP. Eucalyptus grandis Hill ex Maiden e Eucalyptus dunnii Maiden como Fontes de Matéria Prima para Serraria [tese]. Curitiba: Universidade Federal do Paraná; 2000.

Savian JF. Estudo Comparativo da Mineralogia Magnética no Solo e em Insetos Sociais da Mata Santa Genebra, Estado de São Paulo [dissertação]. São Paulo: Instituto de Astronomia, Geofísica e Ciências Atmosféricas; 2009.

Wajnberg E, Acosta-Avalos D, Alves OC, Oliveira JF, Srygley Rb, Esquivel DMS. Magnetoreception in eusocial insects: an update. Journal of the Royal Society Interface 2010; 7(Suppl 2): S207-S225. Available from: http://rsif. royalsocietypublishing.org/content/7/Suppl_2/S207. short. PMid:20106876 PMCid:PMC2843992. http:// dx.doi.org/10.1098/rsif.2009.0526.focus 